\title{
Variables affecting the potential efficacy of PRP in providing chronic pain relief
}

\section{Damien P Kuffler}

Institute of Neurobiology, Medical Sciences Campus, University of Puerto Rico, San Juan, PR, USA
Correspondence: Damien P Kuffler Institute of Neurobiology, Medical Sciences Campus, University of Puerto Rico, 20I Blvd del Valle, San Juan, PR 0090I, USA

Tel +I 787 72I 0778

Email dkuffler@hotmail.com
This article was published in the following Dove Medical Press journal: Journal of Pain Research

\begin{abstract}
Although chronic pain affects about 1\% of the US population, it remains largely resistant to treatment. Despite great variability in pain outcomes, the application of autologous platelet-rich plasma (PRP) has become increasingly popular in attempts to reduce chronic pain. The variability in PRP efficacy raises the question of whether PRP actually has an analgesic capacity, and if so, can that capacity be made consistent and maximized. The best explanation for the variability in PRP analgesic efficacy is the failure during PRP preparation and application to take into account variables that can increase or eliminate its analgesic capabilities. This suggests that if the variables are reduced and controlled, a PRP preparation and application protocol can be developed leading to PRP inducing reliable, complete, and long-term pain relief. The goal of this study was to examine some of the variables that influence platelets and see how they might be controlled to increase the analgesic potential of PRP. Among the variables examined are the physiological status of the patient, methods used to prepare PRP, and methods of PRP application. The goal of modifying these variables is to minimize platelet serotonin content, maximize platelet content of factors that reduce inflammation and pain, while maintaining their bioactivity, maximize platelet capacity to aggregate at injury sites, induce rapid and simultaneous release of their contents, and optimize PRP application protocols. It is concluded that controlling some or many of these variables will lead to PRP that induces reliable, maximum, and long-term relief of chronic pain.
\end{abstract}

Keywords: analgesia, anti-inflammation, chronic pain, cytokines, inflammation, nerve trauma, neuropathic pain, platelet-rich plasma, pro-inflammation

\section{Introduction}

About $1 \%$ of the US population suffers from chronic pain that is often severely debilitating and largely resistant to treatment. ${ }^{1}$ Although chronic pain is a major public problem, there is little available effective pain management, and there is still a lack of understanding of the fundamental mechanisms of pain and how pain can be reliably and safely reduced or eliminated. This paper looks at the application of platelet-rich plasma (PRP) to pain sites as a potential technique for reducing or eliminating chronic pain, and the variables that must be addressed if PRP is to achieve its potential of providing some to complete and permanent relief of chronic pain.

Platelets within PRP release a host of pro- and anti-inflammatory mediators that not only induce pain but also reduce inflammation and pain. These factors also alter the wound environment leading to tissue healing and regeneration via the complex effects of regulating stem cell in-migration, proliferation, differentiation, ${ }^{2}$ and anabolic/ catabolic processes. ${ }^{3}$ These multiple functions have led to the use of PRP to stimulate the recovery of nonhealing injuries, typically associated with chronic pain, in fields

Journal of Pain Research 2019:12 109-116

109 
including ear, nose, and throat, orthopedics, sports medicine, dentistry, neurosurgery, ophthalmology, urology, wound healing, cosmetic, cardiothoracic, and maxillofacial surgery., ${ }^{4,5}$

A number of clinical studies concluded that PRP provides little or no pain relief for tendinosis ${ }^{6}$ or rotator cuff tears..$^{6-8}$ However, other clinical studies found that PRP reduces or eliminates pain, such as that associated with tendinosis, ${ }^{9-11}$ rotator cuff tears, ${ }^{12,13}$ osteoarthritis, ${ }^{14}$ plantar fasciitis, ${ }^{15}$ and muscle injuries. ${ }^{16} \mathrm{PRP}$ is also reported to act as an analgesic in animal pain models, such as rat tendinosis, ${ }^{17}$ rotator cuff injury, ${ }^{18}$ and dog tendon injury. ${ }^{19}$ In general, the findings of PRP analgesic influence led to the conclusion that although there are suggestions that PRP can act as an analgesic, the variability in study outcomes resulted from their small study sample sizes and the inclusion of too many variables, which did not allow the studies to be compared, or that the comparative analyses of the outcomes of different studies do not provide sufficient statistical support for the conclusion that PRP acts as an analgesic. ${ }^{4}$

Two major variables proposed to account for the observed differences in PRP efficacy are PRP composition, which differs greatly depending on the device used to prepare PRP, and the method of PRP application..$^{20}$ This has led to the suggestion that consistent analgesic results will only be seen once the variables associated with PRP preparation and application are reduced..$^{20-24}$

However, other variables must also be considered, each of which can exert significant influences on PRP composition and efficacy such as the: 1) physiological status of the individual from whom blood is drawn and on whom it will be applied; 2) platelet activation methods; and 3) types of tissues to which PRP is applied and injury type. These and other variables lead to $>10$-fold differences in platelet concentration within PRP, large differences in the type and concentration of factors within platelets, determine whether platelets aggregate and release their contents at the pain site, while also influences the concentration of factor released at the pain site, the rate of factor release, ratios of the released factors, and bioactivity levels of those factors. While any one variable can significantly alter PRP composition, multiple variables exert greater influences and can entirely block the analgesic efficacy of PRP.

One approach to developing a PRP preparation and application protocol that induces reliable, complete, and permanent pain relief is to determine the common variables associated with studies in which PRP induced good analgesia, and then perform new studies using those common variables. However, other variables that have so far not been considered must be taken into account, and adjusted to maximize the level of PRP-induced pain relief. This paper examines the influences of a number of the most salient variables associated with an individual's physiological status, and methods for preparing and applying PRP, that affect the concentration of platelets in PRP, platelet factor content, concentration, bioactivity, and ratios, and how these variables can be adjusted to optimize the potential analgesic influence of PRP.

\section{Whole blood platelet count}

Platelet count in the whole blood of about $95 \%$ of healthy individuals is between 150,000 and 450,000 per $\mu \mathrm{L}$, with the remainder having higher or lower counts. ${ }^{25}$ Thus, the platelet count in normal patients varies by 3 -fold. Because PRP separation devices are designed to concentrate platelets by a specific amount, the PRP platelet concentration will consequently vary by up to 3-fold depending on the device used. This will have a significant influence on the concentration of factors released from the platelets within the PRP.

\section{Blood pressure}

High blood pressure induces platelets to release their factors into the blood plasma ${ }^{26}$ and can decrease platelet number. ${ }^{27}$ Therefore, prior to PRP preparation, it may be advantageous to lower a patient's blood pressure, if only briefly, thereby allowing platelets to increase in number and recover their normal factor content.

\section{Mental and physical stress}

Mental stress, including mental math and public speaking, and physical stress, such as exercise or surgery, induce platelets to release their bioactive compounds. ${ }^{28}$ Physical stress also induces a surge in blood hormone levels, such as adrenalin, noradrenaline, and serotonin, which increase platelet sensitivity to thrombin, aggregation, and factor release. ${ }^{29}$ Reducing mental and physical stress prior to preparing PRP should result in PRP with a full complement of platelet bioactive compounds, and platelets that are not preinduced to aggregate.

\section{Diet}

Diet can significantly alter platelets properties, especially when diets high in saturated fats, ${ }^{30}$ excessive sugar, ${ }^{31}$ or simple carbohydrates, ${ }^{30}$ by inducing and increasing platelet aggregation. Diets containing caffeine, common in coffee, tea, sodas, cola drinks, "energy" drinks, and chocolate, ${ }^{32}$ quercetin, a flavonoid present in high levels in onions, apples, tea, and wine, ${ }^{33}$ and isoflavones, present in legumes, such as soybeans and chick peas ${ }^{34}$ reduce platelet activation. The 
consumption of anthocyanins, present in many fruits, causes a significant decrease in the number of activated platelets. ${ }^{35}$ Therefore, before applying PRP, a patient's diet should be evaluated and adjusted appropriately to yield PRP with improved potential analgesic efficacy.

\section{Drugs and other consumables}

Increasing levels of alcohol consumption are associated with decreased platelet activation and aggregation, in men more than women, ${ }^{36}$ and reduced platelet responses to thrombin ${ }^{37}$ and collagen. ${ }^{38}$ Limited smoking (three cigarettes/day) increases platelet aggregation. ${ }^{39,40}$ Therefore, reducing or eliminating alcohol and tobacco consumption prior to preparing PRP may allow platelets to reestablish their normal factor content and aggregation properties.

Platelet content is also affected by various drugs and drug therapies, such as selective serotonin reuptake inhibitors (SSRIs), nonsteroidal anti-inflammatory drugs, antihistamines, antibiotics, and tricyclic antidepressants. These drugs suppress normal platelet functions, factor content, and their ability to aggregate, ${ }^{41,42}$ while quinine reduces platelet count.

The importance of serotonin, and specifically plateletreleased serotonin, in inflammatory pain states is indicated by the finding that inflammation is associated with increased endogenous peripheral serotonin levels. ${ }^{43}$ The application of serotonin to nociceptive dorsal root ganglion neurons induces long-term neuron excitation and sensitization during inflammation, ${ }^{44}$ indicating that serotonin directly triggers pain. ${ }^{45}$ Platelets that aggregate at injured nociceptive neurons are the primary source of serotonin activating these neurons. ${ }^{46}$ The nociceptive influence of platelet-released serotonin at injury sites is increased by platelet-released serotonin inducing more platelet recruitment and aggregation, ${ }^{47}$ which continues in a cyclical fashion, while also blocking serotonin reuptake, which further increases nociceptive neuron excitation. ${ }^{47}$

These findings suggest that it may be possible to reduce platelet-induced pain by reducing platelet serotonin content prior to preparing PRP. This can be done by activating platelet delta granules with nitroglycerin, ${ }^{48}$ or 3,4-methylenedioxymethamphetamine. ${ }^{49}$ SSRIs also reduce neuron ${ }^{47,50}$ and platelet ${ }^{51}$ serotonin content by blocking the serotonin transporter, which is responsible for serotonin uptake and accumulation. The potential efficacy of reducing platelet serotonin content as a means of reducing pain is suggested by the finding that individuals on SSRIs show reduced pain due to multiple causes. ${ }^{52}$

The influence of SSRIs in reducing platelet serotonin content and production is short-lived, and shortly after stopping SSRI administration, platelet serotonin content returns to normal. ${ }^{53}$ This suggests that the short-term administration of SSRIs to healthy individuals may be beneficial in reducing pain by reducing platelet serotonin content. Therefore, it should be possible to increase PRP analgesic efficacy by: 1) inhibiting platelet serotonin release during platelet aggregation; ${ }^{54}$ 2) reducing platelet serotonin content, thereby reducing the amount of platelet serotonin available for release ${ }^{47,54}$ and 3 ) reducing platelet aggregation, ${ }^{47}$ thereby further reducing the amount of serotonin available for release.

Additional approaches to reducing pain induction by modulating platelet content include: 1) reducing platelet release of proinflammatory cytokines, such as interferon- $\gamma$ and tumor necrosis factor- $\alpha^{55}$ and 2) increasing the release of endogenous platelet anti-inflammatory cytokines, such as IL-1 receptor antagonist and IL-10, ${ }^{56}$ which promote analgesia.

\section{PRP preparation techniques}

PRP prepared from the whole blood of the same apparently normal healthy individual using the same separation device can vary greatly in the concentration of platelets and other cells, as well as both the composition and concentration of platelet-released factor. ${ }^{57,58}$ Different PRP preparation devices yield PRP with platelet concentrations varying from 1.99- to 9.3-fold over baseline (Arthrex ACP Double Syringe System [Arthrex Inc, Naples, FL, USA] and Biomet Biologics GPS System [Biomet Biologics LLC, Warsaw, IN, USA], respectively). However, the optimal concentration of platelets in PRP that induces maximal pain relief is unknown.

One clinical study reported that PRP with a platelet concentration of 4 - to 5-fold above average baseline count (varying from 1.5 and $3 \times 10^{5} / \mu \mathrm{L}$ ) enhanced peri-implant bone regeneration, while both lower and higher concentrations had inhibitory effects. ${ }^{59}$ A study on the rat tendinopathy model found that PRP with a platelet concentration of $1.0 \times 10^{6}$ / $\mu \mathrm{L}$ induced complete pain relief, whereas PRP with half the platelet concentration induced significantly less pain relief. ${ }^{17}$ However, knowing what concentration of platelets in PRP induces the maximum analgesic effect requires understanding more than just the PRP platelet concentration. First, PRP derived from different devices and with the same platelet concentration yield greatly differing concentrations of platelet-released factors. ${ }^{60}$ Second, the concentration of platelet-released factors from PRP is not well correlated with platelet count in whole blood. ${ }^{61}$ Third, the concentration of platelet-released factors does not increase in proportion to increasing platelet concentration in PRP. ${ }^{61,62}$ Fourth, some PRP preparation devices reduce the biological activity of platelet-released factors. ${ }^{63}$ 
Different PRP preparation and application techniques use unactivated or activated platelets, and both PRP formulations provide pain relief. However, no quantitative comparative studies have been performed to determine whether one or the other is more efficacious than the other.

Different formulations of platelet-enriched blood products are routinely used. These may be referred to as singlespin, double-spin (or discontinuous cell separation), and the so-called "buffy coat" method. Although the efficacy of each of these has not been compared quantitatively, plateletenriched plasma, obtained after a single-spin procedure, and PRP obtained from a double-spin procedure have been shown to induce similar outcomes when applied to patients with cartilage degenerative lesions and osteoarthritis. ${ }^{64}$

\section{Glucose}

Different PRP separation techniques yield PRP with glucose concentrations increased by 3- to 6-fold over the starting blood. ${ }^{20}$ This increase can significantly affect PRP efficacy because platelet activation increases with increasing glucose concentrations, ${ }^{65}$ and maximum PRP efficacy may require a high ratio of unactivated to activated platelets.

Different separation devices yield PRP with different $\mathrm{pH} .{ }^{20}$ While normal blood $\mathrm{pH}$ is 7.35 , some PRP preparation devices use acidic citrate as an anticoagulant to prevent platelet activation, which results in platelet acidification with approximate $\mathrm{pH}$ values of $6.5-6.8{ }^{66}$ Acidification reduces platelet aggregation by $>50 \% .{ }^{67}$ The subsequent use of thrombin on these platelets within PRP to induce fibrin polymerization during the PRP preparation induces the rapid elevation of platelet intracellular calcium and increases hydrogen ion production causing further platelet acidification, ${ }^{68}$ thereby further reducing platelet aggregation. Alternatives to acidification may be needed, such as preincubating PRP in a more alkaline $\mathrm{pH}$ so that the lysed platelet concentrates contain greater concentrations of factors that stimulate anti-inflammatory actions and pain relief.

\section{PRP leukocyte count}

The concentration of leukocytes in PRP is important to its analgesic efficacy. This is because leukocyte-rich PRP (L-PRP) contains elevated concentrations of inflammatory $^{69}$ and catabolic cytokines ${ }^{70}$ that cause inflammation and apoptosis, thereby reducing the ability of PRP to reduce inflammation, pain, and tissue healing. PRP leukocyte count is dependent on the PRP separation device with leukocyte concentrations varying from 0.13 - to 5-fold over whole blood. ${ }^{20}$ Although the injection of L-PRP has been reported to be beneficial in reducing tendinopathy ${ }^{71} \mathrm{PRP}$ with a reduced leukocyte concentration has also been reported to increase the capacity of PRP to reduce pain. ${ }^{17}$ However, while the leukocyte concentration is influenced by the PRP preparation device, it is also influenced by leukocyte levels in whole blood, with predictors of significantly elevated leukocyte counts including young age, male sex, increased body mass index, smoking, and low alcohol consumption. ${ }^{72}$ Physiological stress and injury also significantly increase blood leukocyte count, due to their increasing blood corticosteroid levels. ${ }^{73}$ Thus, differences in leukocyte concentrations due to physiology and the type of PRP separation device used may also account for some of the variability seen in PRP analgesic efficacy. ${ }^{9}$

\section{Platelet activation methods}

For the factors within platelet alpha granules to be released and effective, the platelets must be activated, such as by thrombin, calcium chloride, or collagen type I. Each activation method results in different rates of platelet clotting, type, amount, and timing of factor release. ${ }^{74}$ These variations can result in major differences in PRP efficacy due to when specific released factors reach their maximum concentration, which factors are available to act simultaneously, the ratios of those factors, and how long the factors are present to act on tissues. Also, having an impact of how these factors influence tissues is that many released factors have a short half-life (from minutes to hours) and, if they are not immediately active when released, they may be degraded before exerting an influence. ${ }^{75}$

Thrombin, in a dose-dependent manner, induces the most reliable, fastest, and most largest release of platelet factors. ${ }^{57}$ However, a 12-fold increase in thrombin concentration does not induce an increase in the release of platelet factors ${ }^{63}$ Calcium chloride induces slower, more prolonged, and overall less factor release. Collagen type I induces lower overall factor release. ${ }^{76}$ It has been proposed that the rapid release of platelet factors may be beneficial for inducing an anti-inflammatory effect. ${ }^{76}$

\section{PRP application guidance techniques}

Differences in PRP pain relief efficacy appear in part due differences in the precision of PRP application to the pain site. This is supported by the finding that, compared to blind injections, greater pain suppression rates are obtained using image guidance tools, such as MRI, ${ }^{23}$ dynamic fluoroscopic control, ${ }^{77}$ and ultrasound, ${ }^{21}$ with ultrasound being the most convenient and reliable. 


\section{Local anesthetics during PRP injection}

Local anesthetics are sometime given prior to injections to reduce pain associated with PRP injections. However, although local anesthetics significantly decrease platelet function, assessed by their capacity to aggregate, ${ }^{78}$ they do not appear to interfere with the platelet growth factor release. ${ }^{78}$ The use of local anesthetics needs to be further examined.

\section{Timing of PRP administration}

PRP is normally injected into a painful injury site several weeks postinjury once the pain is considered chronic. For rats, the optimal time for PRP administration is immediately postinjury. ${ }^{79}$ There is no information about the optimal time to apply PRP clinically.

\section{Single vs multiple injections}

The efficacy of multiple PRP injections in reducing pain varies between studies. For tendinopathy, increasing PRP applications from once to twice at 7 and 14 days postsurgery did not provide additional analgesic benefits, ${ }^{80}$ while two PRP injections 2 weeks apart were more effective than one ${ }^{81}$ For early state osteoarthritis, multiple PRP injections induce better results than a single PRP, but not for advanced osteoarthritis. ${ }^{82}$ However, in another study, patients suffering from early stage osteoarthritis benefitted as well from one injection as two injections 3 weeks apart, but for patients with grade 3 osteoarthritis, a minimum of two PRP injections is required to significantly reduce the pain. ${ }^{83}$ While the efficacy of single vs multiple PRP injections in reducing pain due to osteoarthritis appears to be related to the stage of the disease, optimizing the efficacy of PRP injections requires further studies to determine the best timing and frequency of injections.

\section{Variable PRP action in the same individual}

While the variables discussed above can play important roles for potential PRP efficacy, the importance of other unknown variables in influencing PRP efficacy is demonstrated by the case of a patient whose PRP efficacy is seen in the case of a patient whose PRP was prepared and administered identically on two occasions. The patient suffered from severe chronic pain associated with rotator cuff tears (RCTs) in one shoulder, which was rapidly and permanently eliminated by an injection of PRP. However, when 7 months later similar pain developed in the contralateral shoulder due to RCTs, an injection of PRP had no influence on the pain. ${ }^{12}$ Thus, despite no apparent variables in the patient, PRP preparation, or application, PRP may or may not be effective as an analgesic. This result suggests that very subtle, and yet to be understood, variables can significantly alter PRP composition and its efficacies.

\section{Discussion}

Some clinical studies report that PRP provides little or no clinical pain relief, ${ }^{6-8}$ and these conclusions are supported by meta-analyses of similar clinical studies. ${ }^{7,84,85}$ However, other clinical studies report that PRP induces pain reduction/elimination, ${ }^{9-16,86-89}$ and these reports are supported by meta-analyses of many studies. ${ }^{71,90,91}$ In general, these latter studies conclude that, although the data suggest that PRP has an analgesic effect, valid comparative analysis of the data is not possible due to the small study sample sizes, data being anecdotal, and too many variables being associated with how the PRP was prepared and applied. ${ }^{92}$

Simply performing similar new larger controlled studies will not resolve the question of whether PRP can reliably act as an effective analgesic. This is because the outcomes of studies involving only one variable, such as different PRP separation devices that yield PRP with up to almost a 10 -fold difference in platelet concentration, will yield different results. Consistent results are even less likely when studies involve multiple variables that include: 1) a 3-fold difference in platelet count in whole blood of normal healthy individuals; 2) whole blood platelet count and platelet factor varying several fold due to differences in individuals' physiological status, such as illness, physical and mental stress, and alcohol and drug consumption; 3) varying ratios of cells, such as leukocytes and platelets in PRP, differences in PRP pH from 7.35 to 6.5, 3- to 6-fold variations of glucose concentration; 4) significant differences in how PRP is prepared and platelets are activated, resulting in different ratios of unactivated to activated platelets, differences in the concentrations and the ratios of factors, whether all the released factors are bioactive, and whether they act at the same time; 5) different PRP application techniques, such as when PRP is applied postinjury, the number of PRP applications, and the time between multiple applications; 6) whether PRP is applied to the same or different types of tissues, with similar or different causes of trauma; and 7) other poorly understood or unknown variables.

The findings that PRP can act as an analgesic suggest that PRP has this capacity despite the many variables that can negatively influence platelets. At the same time, the greatly varied results using many different protocols indicate that definitive proof of PRP analgesic efficacy will only be obtained from new and larger studies involving comparable protocols and minimal other variables. ${ }^{71}$ New studies should be performed using only those variables that have been already seen to 
yield reliable analgesia, although multiple studies may be required involving different protocols. All other variables studies should be noted and controlled if possible. Thus, prior to drawing blood, it will be important to obtain the best understanding of the patient's general physiology, such as wellness and disease levels. Simultaneously, the patient's diet, drugs, and other consumables must be controlled to assure that whole blood has a normal platelet concentration, the platelets have their full complement of factors, and they exhibit a normal ability to aggregate, be activated, and release their factors. Finally, it will be essential to determine whether reducing physical and mental stress, or prescribing SSRIs enhanced the capacity of PRP to act as an analgesic.

This paper has examined some of the variables effecting the composition of PRP and how they may be controlled to obtain PRP with maximum alpha granule factor, but minimum serotonin content, platelets that can aggregate normally, while rapidly and completely releasing their entire factor content leading to PRP with maximum analgesic properties. Understanding which variables must be controlled will lead to the development of a standardized protocol yielding PRP that induces reliable, long-term, and complete pain relief.

\section{Disclosure}

The author reports no conflicts of interest in this work.

\section{References}

1. Moalem G, Tracey DJ. Immune and inflammatory mechanisms in neuropathic pain. Brain Res Rev. 2006;51(2):240-264.

2. Andrae J, Gallini R, Betsholtz C. Role of platelet-derived growth factors in physiology and medicine. Genes Dev. 2008;22(10):1276-1312.

3. Kisiday JD, McIlwraith CW, Rodkey WG, Frisbie DD, Steadman JR. Effects of platelet-rich plasma composition on anabolic and catabolic activities in equine cartilage and meniscal explants. Cartilage. 2012;3(3):245-254.

4. Sampson S, Gerhardt M, Mandelbaum B. Platelet rich plasma injection grafts for musculoskeletal injuries: a review. Curr Rev Musculoskelet Med. 2008;1(3-4):165-174.

5. Tschon M, Fini M, Giardino R, et al. Lights and shadows concerning platelet products for musculoskeletal regeneration. Front Biosci. 2011;3:96-107.

6. Ribeiro AG, Ricioli W, Silva AR, Polesello GC, Guimarães RP. Prp in the treatment of trochanteric syndrome: a pilot study. Acta Ortop Bras. 2016;24(4):208-212.

7. Fu CJ, Sun JB, Bi ZG, Wang XM, Yang CL. Evaluation of plateletrich plasma and fibrin matrix to assist in healing and repair of rotator cuff injuries: a systematic review and meta-analysis. Clin Rehabil. 2017;31(2):158-172.

8. Verhaegen F, Brys P, Debeer P. Rotator cuff healing after needling of a calcific deposit using platelet-rich plasma augmentation: a randomized, prospective clinical trial. J Shoulder Elbow Surg. 2016;25(2):169-173.

9. Fitzpatrick J, Bulsara MK, O'Donnell J, Mccrory PR, Zheng MH. The effectiveness of platelet-rich plasma injections in gluteal tendinopathy: a randomized, double-blind controlled trial comparing a single plateletrich plasma injection with a single corticosteroid injection. Am J Sports Med. 2018;46(4):933-939.

10. Mishra A, Pavelko T. Treatment of chronic elbow tendinosis with buffered platelet-rich plasma. Am J Sports Med. 2006;34(11):1774-1778.
11. Peerbooms JC, Sluimer J, Bruijn DJ, Gosens T. Positive effect of an autologous platelet concentrate in lateral epicondylitis in a double-blind randomized controlled trial: platelet-rich plasma versus corticosteroid injection with a 1-year follow-up. Am J Sports Med. 2010;38(2):255-262.

12. Kuffler DP. Differing efficacies of autologous platelet-rich plasma treatment in reducing pain following rotator-cuff injury in a single patient. J Pain Res. 2018;11:2239-2245.

13. Rha DW, Park GY, Kim YK, Kim MT, Lee SC. Comparison of the therapeutic effects of ultrasound-guided platelet-rich plasma injection and dry needling in rotator cuff disease: a randomized controlled trial. Clin Rehabil. 2013;27(2):113-122.

14. Patel S, Dhillon MS, Aggarwal S, Marwaha N, Jain A. Treatment with platelet-rich plasma is more effective than placebo for knee osteoarthritis: a prospective, double-blind, randomized trial. Am J Sports Med. 2013;41(2):356-364.

15. Wilson JJ, Lee KS, Miller AT, Wang S. Platelet-rich plasma for the treatment of chronic plantar fasciopathy in adults: a case series. Foot Ankle Spec. 2014;7(1):61-67.

16. Rossi LA, Molina Rómoli AR, Bertona Altieri BA, Burgos Flor JA, Scordo WE, Elizondo CM. Does platelet-rich plasma decrease time to return to sports in acute muscle tear? A randomized controlled trial. Knee Surg Sports Traumatol Arthrosc. 2017;25(10):3319-3325.

17. Yoshida M, Funasaki H, Marumo K. Efficacy of autologous leukocyte-reduced platelet-rich plasma therapy for patellar tendinopathy in a rat treadmill model. Muscles Ligaments Tendons $J$. 2016;6(2):205-215.

18. Dolkart O, Chechik O, Zarfati Y, Brosh T, Alhajajra F, Maman E. A single dose of platelet-rich plasma improves the organization and strength of a surgically repaired rotator cuff tendon in rats. Arch Orthop Trauma Surg. 2014;134(9):1271-1277.

19. Cook JL, Smith PA, Bozynski CC, et al. Multiple injections of leukoreduced platelet rich plasma reduce pain and functional impairment in a canine model of ACL and meniscal deficiency. J Orthop Res. 2016;34(4):607-615.

20. Fitzpatrick J, Bulsara MK, McCrory PR, Richardson MD, Zheng MH. Analysis of platelet-rich plasma extraction: variations in platelet and blood components between 4 common commercial kits. Orthop J Sports Med. 2017;5(1):2325967116675272.

21. Berkoff DJ, Miller LE, Block JE. Clinical utility of ultrasound guidance for intra-articular knee injections: a review. Clin Interv Aging. 2012;7:89-95.

22. Burke CJ, Adler RS. Ultrasound-guided percutaneous tendon treatments. AJR Am J Roentgenol. 2016;207(3):495-506.

23. Groom LM, White NA, Adams MN, Barrett JG. Accuracy of open magnetic resonance imaging for guiding injection of the equine deep digital flexor tendon within the hoof. Vet Radiol Ultrasound. 2017;58(6):671-678.

24. Kuffler DP. Platelet-rich plasma promotes axon regeneration, wound healing, and pain reduction: fact or fiction. Mol Neurobiol. 2015;52(2):990-1014.

25. Grozovsky R, Giannini S, Falet H, Hoffmeister KM. Regulating billions of blood platelets: glycans and beyond. Blood. 2015;126(16):1877-1884.

26. Yokogoshi H, Wurtman RJ. Meal composition and plasma amino acid ratios: effect of various proteins or carbohydrates, and of various protein concentrations. Metabolism. 1986;35(9):837-842.

27. Ahmed Y, van Iddekinge B, Paul C, Sullivan HF, Elder MG. Retrospective analysis of platelet numbers and volumes in normal pregnancy and in pre-eclampsia. Br J Obstet Gynaecol. 1993;100(3):216-220.

28. Hüfner K, Koudouovoh-Tripp P, Kandler C, et al. Differential changes in platelet reactivity induced by acute physical compared to persistent mental stress. Physiol Behav. 2015;151:284-291.

29. Wallén NH, Goodall AH, Li N, Hjemdahl P. Activation of haemostasis by exercise, mental stress and adrenaline: effects on platelet sensitivity to thrombin and thrombin generation. Clin Sci. 1999;97(1):27-35.

30. de Lorgeril M, Renaud S, Mamelle N, Salen P, et al. Mediterranean alpha-linolenic acid-rich diet in secondary prevention of coronary heart disease. Lancet. 1994;343(8911):1454-1459. 
31. Sudic D, Razmara M, Forslund M, Ji Q, Hjemdahl P, Li N. High glucose levels enhance platelet activation: involvement of multiple mechanisms. Br J Haematol. 2006;133(3):315-322.

32. Frary CD, Johnson RK, Wang MQ. Food sources and intakes of caffeine in the diets of persons in the United States. J Am Diet Assoc. 2005;105(1):110-113.

33. Hubbard GP, Wolffram S, Lovegrove JA, Gibbins JM. Ingestion of quercetin inhibits platelet aggregation and essential components of the collagen-stimulated platelet activation pathway in humans. $J$ Thromb Haemost. 2004;2(12):2138-2145.

34. Williams JK, Clarkson TB. Dietary soy isoflavones inhibit in-vivo constrictor responses of coronary arteries to collagen-induced platelet activation. Coron Artery Dis. 1998;9(11):759-764.

35. Alvarez-Suarez JM, Giampieri F, Tulipani S, et al. One-month strawberry-rich anthocyanin supplementation ameliorates cardiovascular risk, oxidative stress markers and platelet activation in humans. $J$ Nutr Biochem. 2014;25(3):289-294.

36. Mukamal KJ, Massaro JM, Ault KA, et al. Alcohol consumption and platelet activation and aggregation among women and men: the Framingham Offspring Study. Alcohol Clin Exp Res. 2005;29(10):1906-1912.

37. Olas B, Wachowicz B, Saluk-Juszczak J, Zieliński T. Effect of resveratrol, a natural polyphenolic compound, on platelet activation induced by endotoxin or thrombin. Thromb Res. 2002;107(3-4):141-145.

38. Shen MY, Hsiao G, Liu CL, et al. Inhibitory mechanisms of resveratrol in platelet activation: pivotal roles of $\mathrm{p} 38 \mathrm{MAPK}$ and NO/cyclic GMP. Br J Haematol. 2007;139(3):475-485.

39. Belch JJ, McArdle BM, Burns P, Lowe GD, Forbes CD. The effects of acute smoking on platelet behaviour, fibrinolysis and haemorheology in habitual smokers. Thromb Haemost. 1984;51(1):006-008.

40. Warkentin TE, Kelton JG. Temporal aspects of heparin-induced thrombocytopenia. N Engl J Med. 2001;344(17):1286-1292.

41. Barkin RL, Fawcett J. The management challenges of chronic pain: the role of antidepressants. Am J Ther. 2000;7(1):31-47.

42. George JN. Platelets. Lancet. 2000;355(9214):1531-1539.

43. Ernberg M, Lundeberg T, Kopp S. Pain and allodynia/hyperalgesia induced by intramuscular injection of serotonin in patients with fibromyalgia and healthy individuals. Pain. 2000;85(1-2):31-39.

44. Walters ET. Nociceptors as chronic drivers of pain and hyperreflexia after spinal cord injury: an adaptive-maladaptive hyperfunctional state hypothesis. Front Physiol. 2012;3:309.

45. Odem MA, Bavencoffe AG, Cassidy RM, et al. Isolated nociceptors reveal multiple specializations for generating irregular ongoing activity associated with ongoing pain. Pain. 2018;159(11):2347-2362.

46. Sommer C. Serotonin in pain and analgesia: actions in the periphery. Mol Neurobiol. 2004;30(2):117-126.

47. Bismuth-Evenzal Y, Gonopolsky Y, Gurwitz D, Iancu I, Weizman A, Rehavi M. Decreased serotonin content and reduced agonist-induced aggregation in platelets of patients chronically medicated with SSRI drugs. J Affect Disord. 2012;136(1-2):99-103.

48. Schlienger RG, Meier CR. Effect of selective serotonin reuptake inhibitors on platelet activation: can they prevent acute myocardial infarction? Am J Cardiovasc Drugs. 2003;3(3):149-162.

49. Rudnick G, Wall SC. Non-neurotoxic amphetamine derivatives release serotonin through serotonin transporters. Mol Pharmacol. 1993;43(2):271-276.

50. Hüfner K, Kandler C, Koudouovoh-Tripp P, et al. Bioprofiling of platelets in medicated patients with depression. J Affect Disord. 2015;172:81-88.

51. Brenner B, Harney JT, Ahmed BA, et al. Plasma serotonin levels and the platelet serotonin transporter. J Neurochem. 2007;102(1):206-215.

52. Mika J, Zychowska M, Makuch W, Rojewska E, Przewlocka B. Neuronal and immunological basis of action of antidepressants in chronic pain: clinical and experimental studies. Pharmacol Rep. 2013;65(6):1611-1621.

53. Kenis G, Maes M. Effects of antidepressants on the production of cytokines. Int J Neuropsychopharmacol. 2002;5(4):401-412.
54. Maurer-Spurej E, Pittendreigh C, Solomons K. The influence of selective serotonin reuptake inhibitors on human platelet serotonin. Thromb Haemost. 2004;91(1):119-128.

55. Leonard BE. The immune system, depression and the action of antidepressants. Prog Neuropsychopharmacol Biol Psychiatry. 2001;25(4):767-780.

56. Kessler B, Rinchai D, Kewcharoenwong C, et al. Interleukin 10 inhibits pro-inflammatory cytokine responses and killing of Burkholderia pseudomallei. Sci Rep. 2017;7(1):42791.

57. Huber SC, Cunha Júnior JL, Montalvão S, et al. In vitro study of the role of thrombin in platelet rich plasma (PRP) preparation: utility for gel formation and impact in growth factors release. $J$ Stem Cells Regen Med. 2016;12(1):2-9.

58. Weibrich G, Kleis WK, Hitzler WE, Hafner G. Comparison of the platelet concentrate collection system with the plasma-rich-in-growthfactors kit to produce platelet-rich plasma: a technical report. Int J Oral Maxillofac Implants. 2005;20(1):118-123.

59. Weibrich G, Hansen T, Kleis W, Buch R, Hitzler WE. Effect of platelet concentration in platelet-rich plasma on peri-implant bone regeneration. Bone. 2004;34(4):665-671.

60. Castillo TN, Pouliot MA, Kim HJ, Dragoo JL. Comparison of growth factor and platelet concentration from commercial platelet-rich plasma separation systems. Am J Sports Med. 2011;39(2):266-271.

61. Weibrich G, Kleis WK, Hafner G, Hitzler WE. Growth factor levels in platelet-rich plasma and correlations with donor age, sex, and platelet count. J Craniomaxillofac Surg. 2002;30(2):97-102.

62. Eppley BL, Woodell JE, Higgins J. Platelet quantification and growth factor analysis from platelet-rich plasma: implications for wound healing. Plast Reconstr Surg. 2004;114(6):1502-1508.

63. Perut F, Filardo G, Mariani E, et al. Preparation method and growth factor content of platelet concentrate influence the osteogenic differentiation of bone marrow stromal cells. Cytotherapy. 2013;15(7):830-839.

64. Filardo G, Kon E, Pereira Ruiz MT, et al. Platelet-rich plasma intraarticular injections for cartilage degeneration and osteoarthritis: singleversus double-spinning approach. Knee Surg Sports Traumatol Arthrosc. 2012;20(10):2082-2091

65. Keating FK, Sobel BE, Schneider DJ. Effects of increased concentrations of glucose on platelet reactivity in healthy subjects and in patients with and without diabetes mellitus. Am J Cardiol. 2003;92(11):1362-1365.

66. Patscheke $\mathrm{H}$. Shape and functional properties of human platelets washed with acid citrate. Haemostasis. 1981;10(1):14-27.

67. Engström M, Schött U, Romner B, Reinstrup P. Acidosis impairs the coagulation: a thromboelastographic study. J Trauma. 2006;61(3):624-628.

68. Zavoico GB, Cragoe EJ, Feinstein MB. Regulation of intracellular $\mathrm{pH}$ in human platelets. Effects of thrombin, A23187, and ionomycin and evidence for activation of $\mathrm{Na}+\mathrm{H}+$ exchange and its inhibition by amiloride analogs. J Biol Chem. 1986;261(28):13160-13167.

69. Mccarrel TM, Minas T, Fortier LA. Optimization of leukocyte concentration in platelet-rich plasma for the treatment of tendinopathy. $J$ Bone Joint Surg Am. 2012;94(19):e143:(1-8).

70. Zhang L, Chen S, Chang P, et al. Harmful effects of leukocyte-rich platelet-rich plasma on rabbit tendon stem cells in vitro. Am J Sports Med. 2016;44(8):1941-1951.

71. Fitzpatrick J, Bulsara M, Zheng MH. The effectiveness of platelet-rich plasma in the treatment of tendinopathy: a meta-analysis of randomized controlled clinical trials. Am J Sports Med. 2017;45(1):226-233.

72. Schwartz J, Weiss ST. Host and environmental factors influencing the peripheral blood leukocyte count. Am J Epidemiol. 1991;134(12):1402-1409.

73. Rovlias A, Kotsou S. The blood leukocyte count and its prognostic significance in severe head injury. Surg Neurol. 2001;55(4):190-196.

74. Mazzocca AD, McCarthy MB, Chowaniec DM, et al. Platelet-rich plasma differs according to preparation method and human variability. J Bone Joint Surg Am. 2012;94(4):308-316.

75. Harrison S, Vavken P, Kevy S, Jacobson M, Zurakowski D, Murray MM. Platelet activation by collagen provides sustained release of anabolic cytokines. Am J Sports Med. 2011;39(4):729-734. 
76. Cavallo C, Roffi A, Grigolo B, et al. Platelet-rich plasma: the choice of activation method affects the release of bioactive molecules. Biomed Res Int. 2016;2016(1):1-7.

77. Rastogi AK, Davis KW, Ross A, Rosas HG. Fundamentals of joint injection. AJR Am J Roentgenol. 2016;207(3):484-494.

78. Bausset $\mathrm{O}$, Magalon J, Giraudo L, et al. Impact of local anaesthetics and needle calibres used for painless PRP injections on platelet functionality. Muscles Ligaments Tendons J. 2014;4(1):18-23.

79. Çirci E, Akman YE, Şükür E, Bozkurt ER, Tüzüner T, Öztürkmen Y. Impact of platelet-rich plasma injection timing on healing of Achilles tendon injury in a rat model. Acta Orthop Traumatol Turc. 2016;50(3):366-372.

80. Ebert JR, Wang A, Smith A, et al. A Midterm evaluation of postoperative platelet-rich plasma injections on arthroscopic supraspinatus repair: a randomized controlled trial. Am J Sports Med. 2017;45(13):2965-2974.

81. Zayni R, Thaunat M, Fayard JM, et al. Platelet-rich plasma as a treatment for chronic patellar tendinopathy: comparison of a single versus two consecutive injections. Muscles Ligaments Tendons J. 2015;5(2):92-98.

82. Görmeli G, Görmeli CA, Ataoglu B, Çolak C, Aslantürk O, Ertem K. Multiple PRP injections are more effective than single injections and hyaluronic acid in knees with early osteoarthritis: a randomized, doubleblind, placebo-controlled trial. Knee Surg Sports Traumatol Arthrosc. 2017;25(3):958-965.

83. Kavadar G, Demircioglu DT, Celik MY, Emre TY. Effectiveness of platelet-rich plasma in the treatment of moderate knee osteoarthritis: a randomized prospective study. J Phys Ther Sci. 2015;27(12): 3863-3867.
84. Grassi A, Napoli F, Romandini I, et al. Is platelet-rich plasma (PRP) effective in the treatment of acute muscle injuries? A systematic review and meta-analysis. Sports Med. 2018;48(4):971-989.

85. Khan M, Bedi A. Cochrane in CORR (®): platelet-rich therapies for musculoskeletal soft tissue injuries review. Clin Orthop Relat Res. 2015;473(7):2207-2213.

86. Kim SJ, Kim EK, Kim SJ, Song DH, et al. Effects of bone marrow aspirate concentrate and platelet-rich plasma on patients with partial tear of the rotator cuff tendon. J Orthop Surg Res. 2018;13(1):1.

87. Sanchez M, Guadilla J, Fiz N, Andia I, et al. Ultrasound-guided plateletrich plasma injections for the treatment of osteoarthritis of the hip. Rheumatology. 2012;51(1):144-150.

88. Sengodan VC, Kurian S, Ramasamy R. Treatment of partial rotator cuff tear with ultrasound-guided platelet-rich plasma. J Clin Imaging Sci. 2017;7(1):32.

89. Tahririan MA, Moezi M, Motififard M, Nemati M, Nemati A. Ultrasound guided platelet-rich plasma injection for the treatment of rotator cuff tendinopathy. Adv Biomed Res. 2016;5:200.

90. Chen J, Justicz N, Lee L. Platelet-rich plasma for the treatment of androgenic alopecia: a systematic review. Facial Plast Surg. Epub 2018 Jun 28.

91. Hurley ET, Lim Fat D, Moran CJ, et al. The efficacy of platelet-rich plasma and platelet-rich fibrin in arthroscopic rotator cuff repair: a meta-analysis of randomized controlled trials. Am J Sports Med. 2018;363546517751397.

92. Martinez-Zapata MJ, Marti-Carvajal AJ, Sola I. Autologous plateletrich plasma for treating chronic wounds. Cochrane Database Syst Rev. 2016;5:CD006899.
Journal of Pain Research

\section{Publish your work in this journal}

The Journal of Pain Research is an international, peer reviewed, open access, online journal that welcomes laboratory and clinical findings in the fields of pain research and the prevention and management of pain. Original research, reviews, symposium reports, hypothesis formation and commentaries are all considered for publication.

\section{Dovepress}

The manuscript management system is completely online and includes a very quick and fair peer-review system, which is all easy to use. Visit http://www.dovepress.com/testimonials.php to read real quotes from published authors. 